\title{
Falling after epidural analgesia: lessons from obstetric anesthesia
}

\author{
Christian Loubert, MD • Roshan Fernando, MD, BCh
}

Received: 12 October 2015/Revised: 10 January 2016/Accepted: 26 January 2016/Published online: 1 February 2016

(c) Canadian Anesthesiologists' Society 2016

Most upper abdominal and thoracic surgeries produce significant postoperative pain, and patients expect anesthesiologists to provide efficacious postoperative pain relief. Modern epidural analgesia provides significant advantages over opioid-based analgesia, including better pain control at rest as well as during mobilization and coughing; improved cardiovascular, respiratory, and gastrointestinal morbidity endpoints; reduced postoperative mortality; less sedation; and less nausea and vomiting. ${ }^{1,2}$ Concerns remain, however, that epidurals may cause lower limb motor weakness and affect proprioception of the legs, which, together with sympathetic blockade-induced postural hypotension, may increase the risk of inpatient postoperative falls. In this month's issue of the Journal, Elsharydah et al. ${ }^{3}$ use the Nationwide Inpatient Sample (NIS) datasets to explore the relationship between epidural analgesia in patients undergoing various thoracic or upper abdominal surgeries and the rate of inpatient accidents and falls (IAF). The authors found that, although the rate of inpatient falls increased over a five-year period, postoperative epidural analgesia (thoracic or lumbar) was not associated with the incidence of IAFs. These results are pertinent because they can provide clinicians with further reassurance about the safety of postoperative epidural analgesia in this setting. Nevertheless, the NIS datasets reviewed by the

\section{Loubert, MD}

Department of Anesthesiology, Hôpital Maisonneuve-Rosemont, Montreal, QC, Canada

\section{R. Fernando, MD, BCh ( $\square)$}

University College London Hospitals, 235 Euston Road, London

NW1 2BU, UK

e-mail: r.fernando@btinternet.com;

roshanagfernando@gmail.com investigators did not provide details about the type, loading dose, and rate of administration of the local anesthetics and opioids used. Furthermore, they did not provide information about the duration of postoperative epidural drug administration and especially any temporal relationship between cessation of the epidural analgesic regimen and the documented fall. The question then arises regarding which epidural regimen can provide pain-free postoperative analgesia for patients while allowing them to walk safely without any increased risk of falling.

The greatest insights into providing ambulatory epidural analgesia arose in the early 1990s from the frequent requests by pregnant women wanting to walk during labour. Lowdose epidural mixtures of local anesthetics with opioids were originally developed to reduce the incidence of epiduralrelated side effects, such as lower limb motor block, and to improve the safety profile of the epidural in case of accidental intravenous or spinal injection. Nevertheless, obstetric anesthesiologists quickly realized that patients could also walk with such epidurals. Over several decades, strategies have been developed to help evolve lumbar epidural analgesia into what is now well known as the "mobile" or "walking epidural". The success of the lowdose / mobile epidural focuses on one basic principle, i.e., reducing the total dose of local anesthetic infused into the epidural space in order to target only the small myelinated Adelta fibres and unmyelinated $\mathrm{C}$ fibres while sparing the larger and myelinated A-alpha motor fibres.

It is quite clear from reviews on modern neuraxial labour analgesia that such advances were made possible using the following four strategies: ${ }^{4,5}$

1. Use of low local anesthetic concentrations.

2. Concomitant use of adjuvants, such as lipophilic opioids, which have a proven local anesthetic-sparing effect. 
3. Tailoring the amount of epidural drug delivered to the parturient's needs using a patient-controlled epidural analgesia (PCEA) regimen.

4. Use of a programmed intermittent epidural bolus (PIEB) drug delivery system rather than a continuous infusion of epidural solution.

The rationale behind the use of PCEA with PIEB follows the idea that driving a high-pressure bolus injection through an epidural catheter will result in better spread of the anesthetic solution in the epidural space than injecting a continuous infusion of the same solution. As hypothesized, several studies have shown a local anesthetic-sparing effect and a reduction in the requirements for manual rescue boluses (physician-delivered) for breakthrough pain during labour when a PCEA with a PIEB regimen was used. ${ }^{6,7}$ Modern epidural analgesia regimens in labour now commonly include low-concentration local anesthetic solutions, such as $0.0625 \%$ bupivacaine or $0.1 \%$ ropivacaine with fentanyl $2-3 \mu \mathrm{g} \cdot \mathrm{mL}^{-1}$, given as a PCEA regimen with a continuous epidural background infusion or a PIEB. In fact, given the great interest in PIEB, manufacturers are progressively marketing infusion pumps that will allow the use of PCEA with a PIEB regimen.

The interest in the "mobile epidural" is not limited to the obstetric population; it can also be used for elective colorectal surgery patients, especially if an Enhanced Recovery After Surgery (ERAS) protocol is applied. An ERAS protocol consists of a series of perioperative evidence-based measures implemented to reduce surgical stress, maintain postoperative physiological function, and enhance mobilization after surgery. ${ }^{8}$ Among these measures, the routine use of mid-thoracic epidural analgesia (TEA) with local anesthetic and low-dose opioids has been advocated as the optimal postoperative analgesic strategy for patients undergoing an open midline laparotomy. ${ }^{8}$ This has been challenged by those who argue that TEA may increase the incidence of postoperative hypotension, lowerlimb motor blockade, and urinary retention, which in turn can delay mobilization and ambulation..$^{9,10}$

The key to improved TEA that is almost devoid of side effects resulting in impaired ambulation and increased risk of falls lies partly within the medical literature. After reviewing studies in several meta-analyses suggesting an increased incidence of hypotension with TEA, we found that, in virtually all of these trials, the investigators used bupivacaine or ropivacaine concentrations that were higher or equal to $0.125 \%$ or $0.2 \%$, respectively. ${ }^{11,12}$ Unfortunately, few studies have investigated the relationship between much lower concentrations of local anesthetics (e.g., "ultra-low-dose" epidurals) and the incidence of lower limb motor block, hypotension, and IAFs in the surgical population. Moreover, studies consistently report a higher incidence of lower limb motor block with lumbar epidurals than with thoracic epidurals. This is supported by Königsrainer et al. in a prospective audit of over 300 patients undergoing thoracoabdominal, upper abdominal, or colorectal surgeries. ${ }^{13}$ The authors showed that a mid- thoracic (T8T11) epidural catheter insertion site was associated with a lower incidence of lower limb motor block compared with a catheter insertion site that depended on the surgical incision level (almost 20\% of these cases were lumbar epidurals). Moreover, the mid-thoracic epidural provided a similar quality of postoperative analgesia.

In one author's (C.L.) institution, a postoperative epidural analgesia protocol integrating the above strategies has been used for many years. This regimen is routinely offered to patients undergoing thoracic, thoracoabdominal, upper abdominal, and colorectal surgeries. For colorectal surgical patients enrolled in an ERAS protocol, an epidural catheter sited at mid-thoracic levels (T6-T10) is used to infuse a PCEA regimen with a continuous background infusion of $0.05 \%$ bupivacaine with fentanyl $2 \mu \mathrm{g} \cdot \mathrm{mL}^{-1}$ and epinephrine $2 \mu \mathrm{g} \cdot \mathrm{mL}^{-1}$. This is typically complemented with adjuvants such as oral or rectal acetaminophen and nonsteroidal anti-inflammatory drugs unless contraindicated. Although this regimen is based on evidence from the obstetric anesthesia literature, information from our prospectively collected ERAS database suggests that, with this TEA regimen, there is a low incidence of lower limb motor blockade $(2.3 \%)$ and inpatient postoperative falls $(0 \%)$.

In conclusion, several strategies that could further reduce the risk of epidural analgesia-associated complications (e.g., hypotension, lower limb motor block, and IAFs) need further investigation in the non-obstetric surgical population. Such strategies include the use of "ultra-low-dose" local anesthetic solutions, the use of adjuvants (e.g., epidural lipophilic opioids), self-adjusted administration of the epidural solution (PCEA), highpressure driven bolus doses (PIEB), and a mid-thoracic (T6-T10) catheter insertion site for all types of surgery. In our view, these are the elements of a modern mobile epidural regimen that would increase the quality and safety of postoperative epidural analgesia.

\section{Chute après analgésie péridurale: leçons de l'anesthésie obstétricale}

La majorité des chirurgies thoraciques et abdominales entraîne une douleur postopératoire significative et les 
patients comptent sur les anesthésiologistes pour leur procurer un soulagement postopératoire efficace de la douleur. L'analgésie péridurale moderne présente des avantages significatifs par rapport à l'analgésie aux opioïdes, notamment meilleur contrôle de la douleur au repos ainsi que pendant la mobilisation et la toux, une amélioration des critères d'évaluation de la morbidité cardiovasculaire, respiratoire et gastro-intestinale, une baisse de la mortalité postopératoire, une sédation moins profonde, et moins de nausées et vomissements. ${ }^{1,2}$ Néanmoins, on craint toujours que la péridurale puisse provoquer une faiblesse des membres inférieurs et altérer la proprioception des jambes ce qui, en combinaison avec l'hypotension posturale induite par le blocage sympathique, peut accroître le risque de chute postopératoire des patients hospitalisés. Dans le numéro de ce mois-ci du Journal, Elsharydah et coll. ${ }^{3}$ utilisent les ensembles de données de la NIS (Nationwide Inpatient Sample) pour explorer les rapports entre l'analgésie péridurale chez des patients subissant différentes chirurgies thoraciques ou abdominales hautes et les taux de chute et d'accidents chez des patients hospitalisés. Les auteurs ont constaté que bien que le taux de chute ait augmenté au cours d'une période de cinq ans, l'analgésie péridurale postopératoire (thoracique ou lombaire) n'était pas associée à l'incidence des chutes et accidents chez les patients hospitalisés. Ces résultats sont pertinents, car ils peuvent rassurer les cliniciens sur l'innocuité de l'analgésie péridurale postopératoire dans ce contexte. Néanmoins, les ensembles de données du NIS analysés par les investigateurs n'ont pas fourni de détails sur le type, la dose de charge et la vitesse d'administration des anesthésiques locaux ainsi que sur les types d'opioïdes utilisés. Ils n'ont pas fourni de renseignements sur la durée d'administration postopératoire du médicament de la péridurale et, plus particulièrement, sur les relations temporelles entre l'arrêt du protocole d'analgésie péridurale et la chute documentée. Il faut alors se poser la question de savoir quels protocoles périduraux peuvent procurer une absence de douleurs par analgésie postopératoire chez les patients tout en leur permettant de marcher sans danger et sans risque accru de chute.

Nos meilleures connaissances sur la réalisation d'une analgésie péridurale ambulatoire datent du début des années 1990 avec la demande fréquente de femmes enceintes désirant marcher pendant la progression du travail. Les mélanges pour péridurale d'anesthésiques locaux à faible dose et d'opioïdes ont été initialement élaborés pour réduire l'incidence des effets secondaires liés à la péridurale, tels qu'un bloc moteur des membres inférieurs, et pour améliorer le profil de sécurité de la péridurale en cas d'injection accidentelle intraveineuse ou médullaire. Néanmoins, les anesthésiologistes obstétricaux ont vite réalisé que les patientes pouvaient également marcher avec de telles péridurales. Des stratégies ont été élaborées au cours de plusieurs décennies pour aider à faire évoluer l'analgésie péridurale lombaire vers ce que nous appelons maintenant communément la «péridurale mobile » ou «péridurale permettant la marche ». Le succès de la péridurale à faible dose/mobile repose sur un principe de base, à savoir réduire la dose totale d'anesthésique local injecté dans l'espace péridural afin de ne cibler que les petites fibres myélinisées A-delta et les fibres non myélinisées $\mathrm{C}$, tout en épargnant les fibres motrices myélinisées A-alpha plus grandes.

Il ressort clairement des analyses de l'analgésie neuraxiale moderne du travail que de tels progrès ont été rendus possibles en utilisant les quatre stratégies suivantes: ${ }^{4,5}$

5. Utilisation de faibles concentrations d'anesthésiques locaux.

6. Utilisation concomitante d'adjuvants tels que des opiö̈des lipophiles qui ont démontré leur effet d'économie des anesthésiques locaux.

7. L'adaptation sur mesure de la quantité de médicaments périduraux administrés pour répondre aux besoins des parturientes en utilisant un protocole d'analgésie péridurale contrôlée par la patiente (APCP).

8. Utilisation d'un système d'administration des médicaments pour des bolus périduraux intermittents programmés (BPIP) plutôt qu'une administration continue de solution péridurale.

La justification de l'utilisation d'une APCP avec BPIP repose sur l'idée que l'injection d'un bolus à haute pression via un cathéter péridural entraînera une meilleure répartition de la solution anesthésique dans l'espace péridural qu'une injection continue de la même solution. Comme le suggérait l'hypothèse, plusieurs études ont montré un effet d'économie des anesthésiques locaux et une réduction de la demande de bolus manuels de secours (administrés par le médecin) contre les poussées douloureuses au cours du travail quand un protocole d'APCP était utilisé avec des BPIP. ${ }^{6,7}$ Les protocoles modernes d'analgésie péridurale au cours du travail incluent maintenant habituellement des solutions d'anesthésiques locaux à faible concentration (par exemple, bupivacaïne à $0,0625 \%$ ou ropivacaïne à $0,1 \%$ ) administrées avec du fentanyl (2 à $\left.3 \mu \mathrm{g} \cdot \mathrm{mL}^{-1}\right)$ en APCP, avec une perfusion péridurale continue de fond ou un BPIP. En fait, compte tenu du grand intérêt pour les BPIP, les fabricants commercialisent progressivement des pompes de perfusion qui permettront l'utilisation de protocoles d'APCP avec des BPIP.

L'intérêt pour la « péridurale mobile » ne se limite pas à la population obstétricale; elle peut aussi être utilisée pour 
les patients subissant une chirurgie colorectale programmée, en particulier quand on prévoit un protocole de Récupération rapide après la chirurgie (RRAC). Un protocole de RRAC consiste en la mise en œuvre d'une série de mesures peropératoires basées sur des données probantes destinées à réduire le stress chirurgical, maintenir les fonctions physiologiques postopératoires et faciliter la mobilisation après l'intervention. ${ }^{8}$ Parmi ces mesures, l'utilisation habituelle de l'analgésie péridurale médiothoracique (TEA) avec un anesthésique local et de faibles doses d'opioïdes a été préconisée comme étant la stratégie analgésique postopératoire optimale chez les patients subissant une laparotomie médiane ouverte. ${ }^{8}$ Cela a été remis en cause par ceux qui affirment que la TEA pourrait augmenter l'incidence des hypotensions postopératoires, des blocs moteurs des membres inférieurs et de la rétention urinaire, événements qui risquent de retarder la mobilisation et la reprise de la marche. ${ }^{9,10}$

L'élément essentiel pour une TEA améliorée pratiquement dénuée d'effets indésirables entraînant un trouble de la marche et une augmentation du risque de chutes se trouve en partie dans la littérature médicale. Après avoir examiné les études dans plusieurs métaanalyses suggérant une augmentation de l'incidence de l'hypotension avec la TEA, nous avons constaté que dans quasiment tous ces essais, les investigateurs avaient utilisé des concentrations de bupivacaïne ou de ropivacaïne qui étaient égales ou supérieures à, respectivement, $0,125 \%$ ou $0,2 \% .^{11,12}$ Malheureusement, peu d'études se sont intéressées aux rapports entre des concentrations d'anesthésiques locaux beaucoup plus faibles (par exemple, les péridurales à « dose ultra basse ») et l'incidence des blocs moteurs des membres inférieurs, l'hypotension et les accidents et chutes des patients hospitalisés dans la population chirurgicale. De plus, les études ont régulièrement signalé une incidence des blocs moteurs des membres inférieurs plus élevée avec les péridurales lombaires qu'avec les péridurales thoraciques. Cela est étayé par une étude prospective de Königsrainer et coll. sur plus de 300 patients ayant subissant une intervention chirurgicale thoraco-abdominale, abdominale haute ou colorectale. ${ }^{13}$ Les auteurs ont montré qu'un site d'insertion médio-thoracique (D8-D11) du cathéter péridural était associé à une moindre incidence des blocs moteurs des membres inférieurs, comparativement à un site d'insertion du cathéter au niveau de l'incision chirurgicale (près de $20 \%$ de ces cas étaient des péridurales lombaires). En outre, une péridurale médio-thoracique produisait une qualité d'analgésique postopératoire similaire.

Dans l'institution de l'un des auteurs (C.L.), un protocole d'analgésie péridurale postopératoire intégrant les stratégies énumérées plus tôt est utilisé depuis plusieurs années. Ce protocole est régulièrement proposé aux patients subissant une intervention chirurgicale thoraco-abdominale, abdominale haute ou colorectale. Pour les patients subissant une chirurgie colorectale incluse dans un protocole de RRAC, un cathéter péridural localisé aux niveaux médio-thoraciques (D6 à D10) sert à perfuser un protocole d'APAP avec une perfusion continue de fond de bupivacaïne à $0,05 \%$ avec fentanyl $2 \mu \mathrm{g} \cdot \mathrm{mL}^{-1}$ et adrénaline $2 \mu \mathrm{g} \cdot \mathrm{mL}^{-1}$. Ce protocole est habituellement complété par un traitement supplémentaire, tel que de l'acétaminophène par voie orale ou rectale et des anti-inflammatoires non stéroïdiens, sauf contre-indications. Bien que ce schéma de traitement repose sur les données probantes issues de la littérature en matière d'anesthésie obstétricale, les renseignements collectés de manière prospective à partir de notre base de données de RRAC suggèrent qu'il n'y a qu'une faible incidence de blocs moteurs des membres inférieurs $(2,3 \%)$ et de chutes postopératoires des patients hospitalisés $(0 \%)$ avec ce protocole de TEA.

En conclusion, plusieurs stratégies susceptibles de réduire encore plus le risque de complications associées à l'analgésie péridurale (par ex., hypotension, bloc moteur des membres inférieurs, accidents et chutes chez les patients hospitalisés) doivent faire l'objet d'études complémentaires dans la population chirurgicale non obstétricale. Ces stratégies incluent l'utilisation de solutions d'anesthésiques locaux à « dose ultra faible », l'utilisation de compléments (par ex., opioïdes lipophiles périduraux), administration autoajustée de la solution péridurale (APAP), doses de bolus sous haute pression (BPIP) et site d'insertion médio-thoracique du cathéter (D6-D10) pour tous les types de chirurgies. Selon nous, ce sont là les éléments d'un protocole moderne de péridurale mobile qui augmenteraient la qualité et l'innocuité de l'analgésie péridurale postopératoire.

Conflicts of interest None declared.

Conflit d'intérêt Aucun.

\section{References}

1. Behera BK, Puri GD, Ghai B. Patient-controlled epidural analgesia with fentanyl and bupivacaine provides better analgesia than intravenous morphine patient-controlled analgesia for early thoracotomy pain. J Postgrad Med 2008; 54: 86-90.

2. Popping DM, Elia N, Van Aken HK, et al. Impact of epidural analgesia on mortality and morbidity after surgery: systematic review and meta-analysis of randomized controlled trials. Ann Surg 2014; 259: 1056-67.

3. Elsharydah A, Williams TM, Rosero EB, Joshi GP. Epidural analgesia does not increase the rate of inpatient falls after major upper abdominal and thoracic surgery: a retrospective case- 
control study. Can J Anesth 2016; 63: this issue. DOI: 10.1007/ s12630-016-0602-5.

4. Loubert C, Hinova A, Fernando R. Update on modern neuraxial analgesia in labour: a review of the literature of the last 5 years. Anaesthesia 2011; 66: 191-212.

5. Stewart A, Fernando R. Maternal ambulation during labor. Curr Opin Anaesthesiol 2011; 24: 268-73.

6. Sia AT, Lim Y, Ocampo C. A comparison of a basal infusion with automated mandatory boluses in parturient-controlled epidural analgesia during labor. Anesth Analg 2007; 104: 673-8.

7. Wong CA, Ratliff JT, Sullivan JT, Scavone BM, Toledo P, McCarthy RJ. A randomized comparison of programmed intermittent epidural bolus with continuous epidural infusion for labor analgesia. Anesth Analg 2006; 102: 904-9.

8. Gustafsson UO, Scott MJ, Schwenk W, et al. Guidelines for perioperative care in elective colonic surgery: Enhanced Recovery After Surgery (ERAS $((\mathrm{R}))$ ) Society recommendations. World J Surg 2013; 37: 259-84.
9. Low J, Johnston N, Morris C. Epidural analgesia: first do no harm. Anaesthesia 2008; 63: 1-3.

10. Patel GN, Rammos CK, Patel JV, Estes NC. Further reduction of hospital stay for laparoscopic colon resection by modifications of the fast-track care plan. Am J Surg 2010; 199: 391-4; discussion 4-5.

11. Baidya DK, Khanna $P$, Maitra $S$. Analgesic efficacy and safety of thoracic paravertebral and epidural analgesia for thoracic surgery: a systematic review and meta-analysis. Interact Cardiovasc Thorac Surg 2014; 18: 626-35.

12. Carrier FM, Turgeon AF, Nicole PC, et al. Effect of epidural analgesia in patients with traumatic rib fractures: a systematic review and meta-analysis of randomized controlled trials. Can $\mathrm{J}$ Anesth 2009; 56: 230-42.

13. Konigsrainer I, Bredanger S, Drewel-Frohnmeyer R, et al. Audit of motor weakness and premature catheter dislodgement after epidural analgesia in major abdominal surgery. Anaesthesia 2009; 64: 27-31. 